\title{
Estilos de vida saludable y rendimiento académico en estudiantes universitarios
}

Healthy lifestyles and academic performance in university students

\author{
JUAN ABEL PALOMINO ORIZANO ${ }^{1}$
}

https://orcid.org/0000-0003-2546-9684

\section{GUDELIA ZEVALLOS YPANAQUÉ 2}

https://orcid.org/0000-0003-4197-6619

\section{LINCOLN ABEL ORIZANO QUEDO}

\author{
https://orcid.org/0000-0002-2613-1901
}

\begin{abstract}
RESUMEN: Es un estudio que tuvo como objetivo identificar la relación entre los estilos de vida saludable y el rendimiento académico en una muestra de 157 estudiantes universitarios de la Facultad de Educación Inicial de la Universidad Nacional de Educación Enrique Guzmán y Valle, Perú. Se realizó mediante diseño descriptivo correlacional transversal. Los datos se recolectaron a través del Cuestionario de prácticas y creencias sobre estilos de vida, tomado de Arrivillaga, Salazar y Gómez (2002) y el rendimiento académico se obtuvo de los Registros de promedios ponderados de rendimiento académico emitidos por la Oficina de Registro y Servicios Académicos de la UNE. Llegando a los siguientes resultados y conclusiones: Un $85.99 \%$ de estudiantes obtuvieron estilos de vida medianamente saludable y un $67.50 \%$ obtuvieron un rendimiento académico promedio o aprendizaje regularmente logrado, lo que indica que la presencia de estilos de vida medianamente saludable en los estudiantes favorece de manera regular su rendimiento académico. También se observó un coeficiente de correlación de Rho de Spearman $(\mathrm{r}=0,6529)$ positiva media entre estilos de vida saludable y rendimiento académico, lo que indica que a mayores niveles de estilos de vida saludable mayores niveles de rendimiento académico o aprendizajes logrados en los estudiantes universitarios.
\end{abstract}

Palabras clave: estilos de vida saludable; estilos de vida; salud; calidad de vida, rendimiento académico.

ABSTRACT: This study aimed to identify the relationship between healthy lifestyles and academic performance in a sample of 157 university students from the Faculty of Early Education at the Enrique Guzmán y Valle National University of Education, Peru. It was 
carried out by means of a cross-sectional descriptive correlation design. The data were collected through the Lifestyle Beliefs and Practices Questionnaire, taken from Arrivillaga, Salazar and Gómez (2002) and the academic performance was obtained from the Weighted Average Records of Academic Performance issued by the UNE's Office of Registration and Academic Services. The following results and conclusions were reached: $85.99 \%$ of students obtained a medium-healthy lifestyle and $67.50 \%$ obtained a medium-healthy academic performance or regularly achieved learning, which indicates that the presence of medium-healthy lifestyles in students regularly favours their academic performance. A mean positive Spearman's Rho correlation coefficient $(r=0.6529)$ was also observed between healthy lifestyles and academic performance, indicating that higher levels of healthy lifestyles lead to higher levels of academic performance or learning achieved by university students.

Keywords: healthy lifestyles; lifestyles; health; quality of life, academic performance.

\section{INTRODUCCIÓN}

En la vida académica de nuestros estudiantes universitarios surgen desequilibrios en cuanto a sus modos y hábitos de vida, lo que repercute indudablemente en su rendimiento académico, en este sentido se quiere demostrar la existencia de una relación entre los estilos de vida saludable y el rendimiento académico.

Hoy en día se observa indicios de sedentarismo, mal uso del tiempo, hábitos alimenticios inadecuados, falta de organización de las actividades, consumo de bebidas alcohólicas y otras sustancias, así como problemas en el sueño; siendo factores que indudablemente afectan al rendimiento académico o aprendizaje de los estudiantes y al mismo tiempo generar problemas de salud como obesidad, desnutrición, diabetes, enfermedades cardiovasculares, hipertensión arterial, osteoporosis, algunos tipos de cáncer y depresión. En este sentido, en esta investigación se planteó como objetivo determinar la relación entre las variables estilos de vida saludable y el rendimiento académico, para lograr este objetivo se delimitó una población de 265 y se determinó aleatoriamente una muestra de 157 estudiantes universitarios de la Facultad de Educación Inicial. Se empleó el método descriptivo de diseño correlacional transeccional. Y se halló como resultado que un $85.99 \%$ de estudiantes en estilos de vida saludable obtuvieron estilos de vida medianamente saludable y un $67.50 \%$ de estudiantes obtuvieron un rendimiento académico promedio o aprendizaje regularmente logrado, lo que indica que la presencia de estilos de vida medianamente saludable en los estudiantes favorece de manera regular su rendimiento académico; también se obtuvo un coeficiente de correlación de Rho de Spearman ( $\mathrm{r}=0,6529)$ positiva media entre estilos de vida saludable y rendimiento académico; concluyendo que si incrementan los estilos de vida saludables incrementa la probabilidad de mejorar el rendimiento académico o aprendizaje en los estudiantes y si los estudiantes practican actividades deportivas, organizan mejor su tiempo, cuidan su salud, mejoran sus hábitos alimenticios y descansan adecuadamente tienden a mejorar sus aprendizajes y rendimiento académico. 


\section{METODOLOGÍA}

Se utilizó el método no experimental o método descriptivo, porque no se realizó ninguna manipulación o control directo de las variables en estudio (Kerlinger \& Lee, 2002) y se aplicó el diseño correlacional transeccional, debido a que solo se llegó a medir y evaluar el grado de relación que existe entre los conceptos o variables Estilos de vida saludable y Rendimiento académico (Hernández et al., 2014, p. 93) en un contexto particular y momento determinado. El instrumento que se empleó para medir la variable estilos de vida saludable fue el Cuestionario de prácticas y creencias sobre estilos de vida, tomado de Arrivillaga et al. (2003) (Arrivillaga \& Salazar, 2005, p. 32), de este instrumento solo se cogió la parte de prácticas y se adaptaron algunos términos de la encuesta, las categorías de calificación del estilo de vida por dimensión fueron: estilo de vida muy saludable (EVMS), estilo de vida saludable (EVS), estilos de vida medianamente saludable (EVM), estilo de vida poco saludable (EVPS) y estilo de vida no saludable (EVNS) (Arrivillaga et al., 2003, p. 189), se halló su validez de contenido mediante juicio de expertos y su confiabilidad se realizó a través de Alfa de Cronbach; y para recoger los datos de la variable rendimiento académico, se hizo uso el Registros de promedios ponderados de rendimiento académico emitidos por la Oficina de Registro y Servicios Académicos de la UNE, las categorías de calificación de rendimiento académico fueron: aprendizaje bien logrado (ABL), aprendizaje regularmente logrado (ARL) y aprendizaje deficiente (ADE) (Reyes, 2003, p.40). La población de estudio estuvo constituida por 265 estudiantes del tercero, quinto, séptimo y noveno ciclo, matriculados en el régimen regular del semestre 2015-I de la Facultad de Educación Inicial de la Universidad Nacional de Educación Enrique Guzmán y Valle, y se determinó la muestra en forma aleatoria haciendo un total de 157 estudiantes, tal como se señala en la tabla 1.

Tabla 1. Población y muestras por estratos

\begin{tabular}{lcc}
\hline Estratos & $\begin{array}{c}\text { Tamaño de pobla- } \\
\text { ción por estratos }\end{array}$ & $\begin{array}{c}\text { Tamaño de la mues- } \\
\text { tra por estratos }\end{array}$ \\
\hline Tercer ciclo & 71 & 42 \\
Quinto ciclo & 59 & 35 \\
Séptimo ciclo & 83 & 49 \\
Noveno ciclo & 52 & 31 \\
\hline Total & $\mathbf{2 6 5}$ & $\mathbf{1 5 7}$ \\
\hline
\end{tabular}

Fuente: estadística de la Oficina de Registro y Servicios Académicos de la UNE

\section{RESULTADOS}

Para tal propósito se analizó los datos obtenidos en el Cuestionario de prácticas y creencias sobre estilos de vida y en los Registros de promedios ponderados de rendimiento académico; de esta manera se sistematizó los resultados a través de las contrastaciones para señalar las relaciones existentes entre estilos de vida saludable y rendimiento académico en dichos estudiantes. El análisis de los datos se realizó mediante el análisis estadístico descriptivo e inferencial, a través del programa STATA-12, obteniendo los siguientes resultados: 
En la figura 1 se muestra los estilos de vida saludable en la dimensión actividad física y deportes de los estudiantes, observándose un mayor porcentaje $(56.1 \%)$ de estudiantes tienen un estilo medianamente saludable, y un menor porcentaje (7\%) de estudiantes tienen un estilo no saludable. Lo que indica que la mayoría de los estudiantes tienen un estilo medianamente saludable en la dimensión actividad física y deportes.

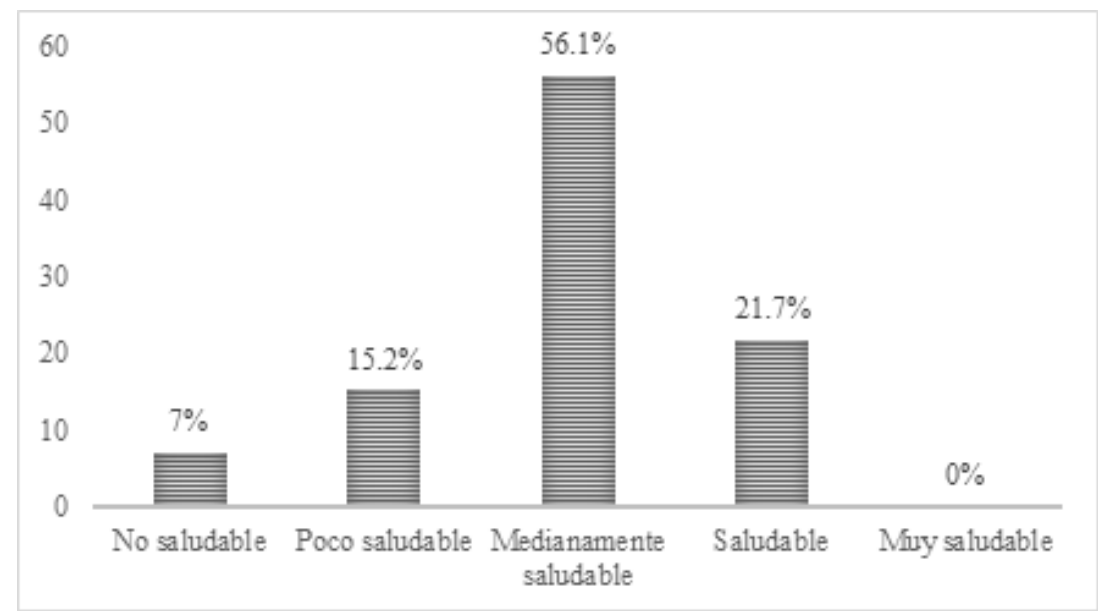

Figura 1. Estilos de vida saludable en la dimensión actividad física y deportes

En la figura 2 se muestra los estilos de vida saludable en la dimensión recreación y manejo del tiempo libre de los estudiantes, observándose un mayor porcentaje (56.7\%) de estudiantes tienen un estilo medianamente saludable, y un menor porcentaje (7\%) de estudiantes tienen estilos no saludables. Lo que indica que la mayoría de los estudiantes tienen un estilo medianamente saludable en la dimensión recreación y manejo del tiempo libre.

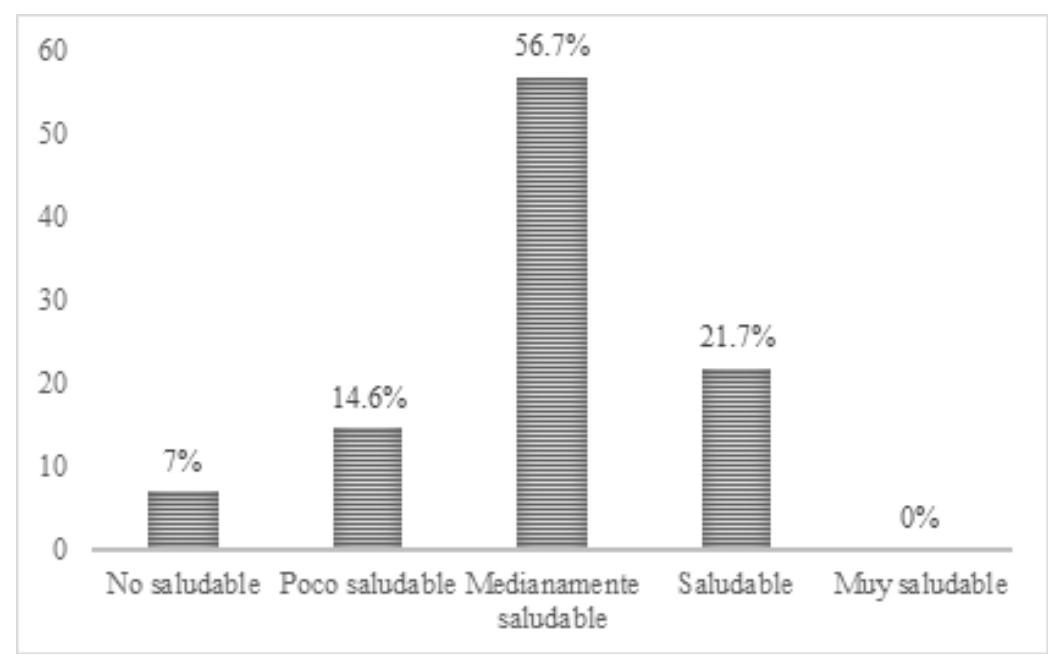

Figura 2. Estilos de vida saludable en la dimensión recreación y manejo del tiempo libre

En la figura 3 se muestra los niveles de estilos de vida saludable en la dimensión autocuidado y cuidado médico de los estudiantes, observándose un mayor porcentaje $(64.3 \%)$ de estudiantes tienen un estilo medianamente saludable, y un menor porcentaje (3.2\%) de estudiantes tiene un estilo no saludable. Lo que indica que la mayoría de los estudiantes tienen un estilo medianamente saludable en la dimensión autocuidado y cuidado médico. 


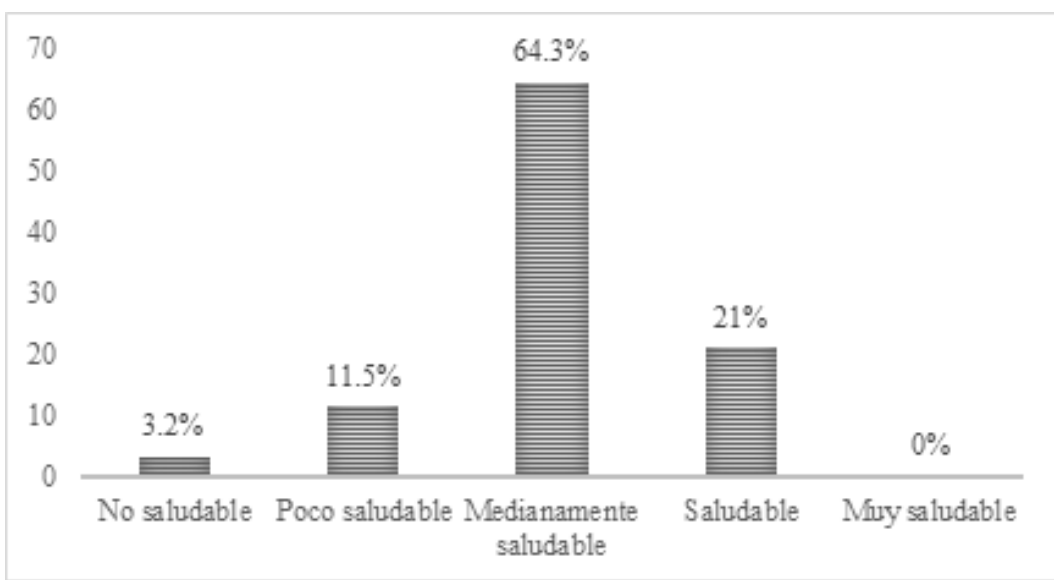

Figura 3. Estilos de vida saludable en la dimensión autocuidado y cuidado médico

En la figura 4 se muestra los niveles de estilos de vida saludable en la dimensión hábitos alimenticios de los estudiantes, observándose un mayor porcentaje $(51 \%)$ de estudiantes tienen un estilo medianamente saludable, y un menor porcentaje $(7 \%)$ de estudiantes tiene un estilo no saludable. Lo que indica que la mayoría de los estudiantes tienen un estilo medianamente saludable en la dimensión hábitos alimenticios.

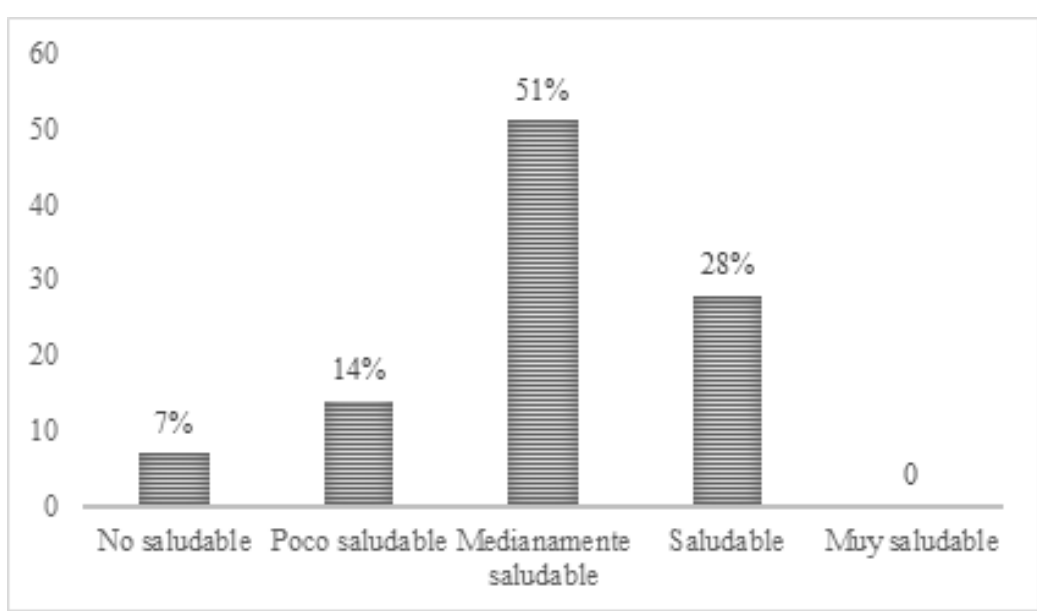

Figura 4. Estilos de vida saludable en la dimensión hábitos alimenticios

En la figura 5 se muestra los niveles de estilos de vida saludable en la dimensión consumo de alcohol, tabaco y otras drogas de los estudiantes, observándose un mayor porcentaje $(46.5 \%)$ de estudiantes tienen un estilo medianamente saludable, y un menor porcentaje $(2.5 \%)$ de estudiantes tiene un estilo no saludable. Lo que indica que la mayoría de los estudiantes tienen un estilo medianamente saludable en la dimensión consumo de alcohol, tabaco y otras drogas. 


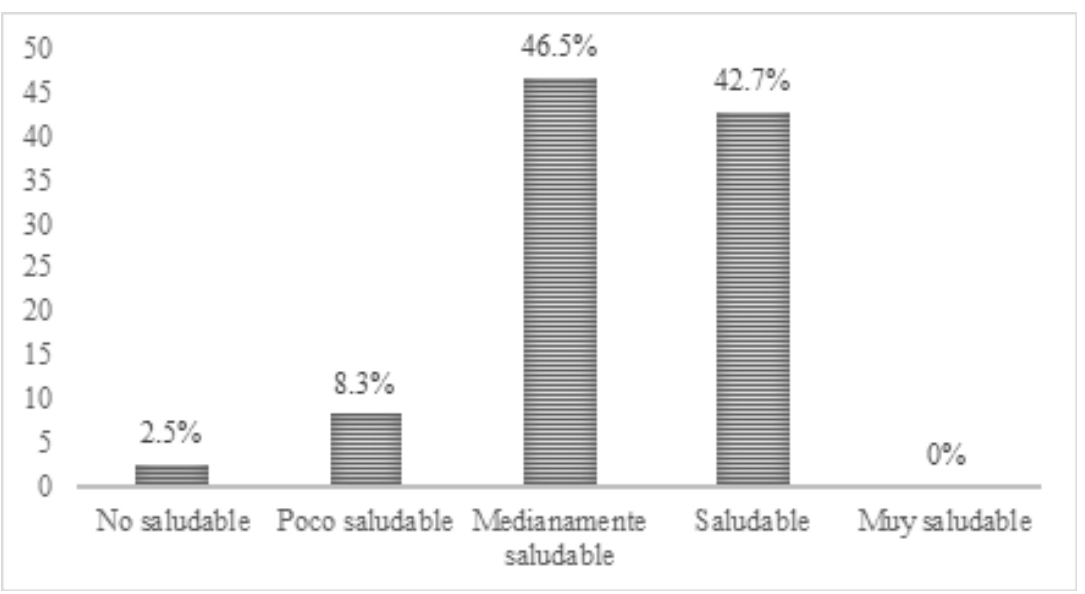

Figura 5. Estilos de vida saludable en la dimensión consumo de alcohol, tabaco y otras drogas

En la figura 6 se muestra los niveles de estilos de vida saludable en la dimensión hábitos de sueño de los estudiantes, observándose un mayor porcentaje (64.3\%) de estudiantes tienen un estilo medianamente saludable, y un menor porcentaje $(2.5 \%)$ de estudiantes tiene un estilo no saludable. Lo que indica que la mayoría de los estudiantes tienen un estilo medianamente saludable en la dimensión hábitos de sueño.

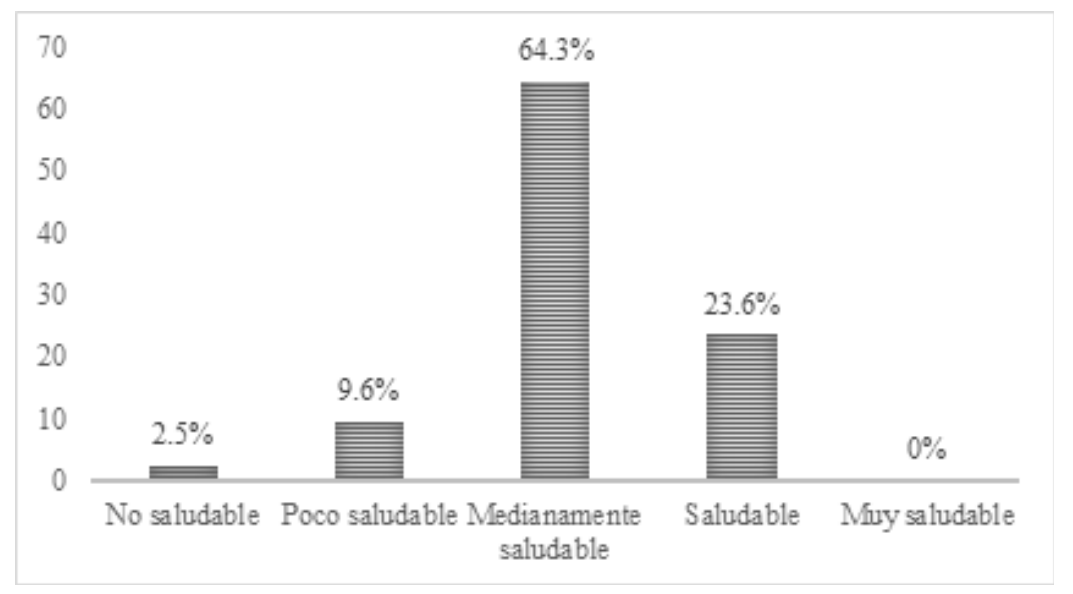

Figura 6. Niveles de estilos de vida saludable en su dimensión hábitos de sueño

En la figura 7 se muestra los estilos de vida saludable en general, observándose que un mayor porcentaje $(85.99 \%)$ de estudiantes tienen un estilo medianamente saludable, y un menor porcentaje $(14.01 \%)$ de estudiantes tienen un estilo saludable. Lo que indica que la mayoría de los estudiantes tienen un estilo medianamente saludable en general. 


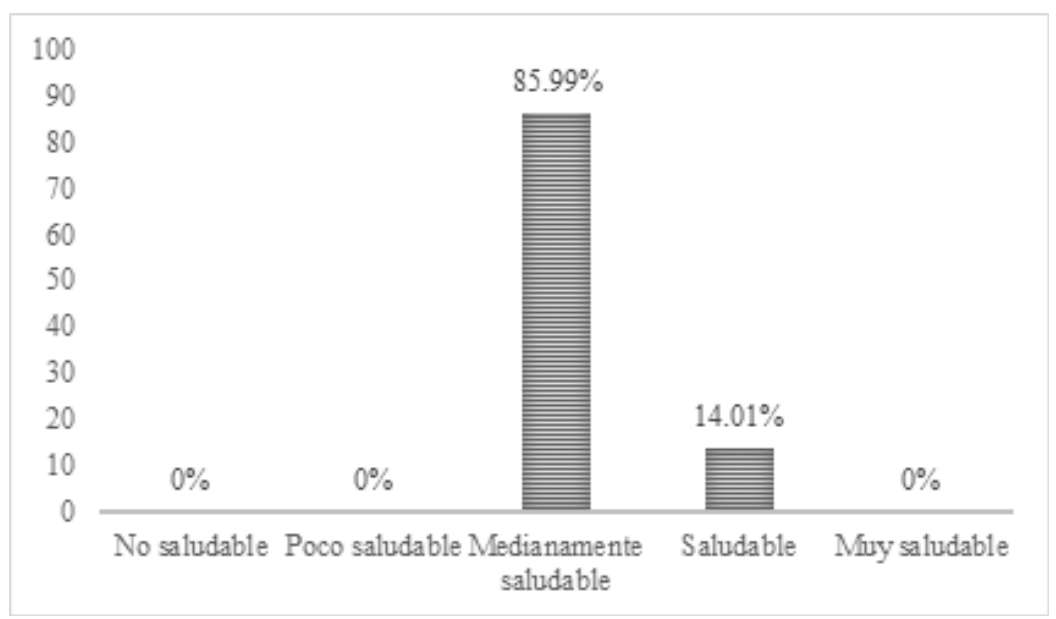

Figura 7. Niveles de estilos de vida saludable en general

En la figura 8 se muestra los niveles de rendimiento académico en general, observándose que un mayor porcentaje $(67.50 \%)$ de estudiantes se encuentran en un nivel promedio en rendimiento académico o aprendizaje regular logrado, y un menor porcentaje $(9.60 \%)$ se encuentran en un nivel bajo en rendimiento académico o aprendizaje deficiente. Lo que indica que la mayoría de estudiantes se encuentran en un nivel promedio en rendimiento académico en general o aprendizaje regular logrado.

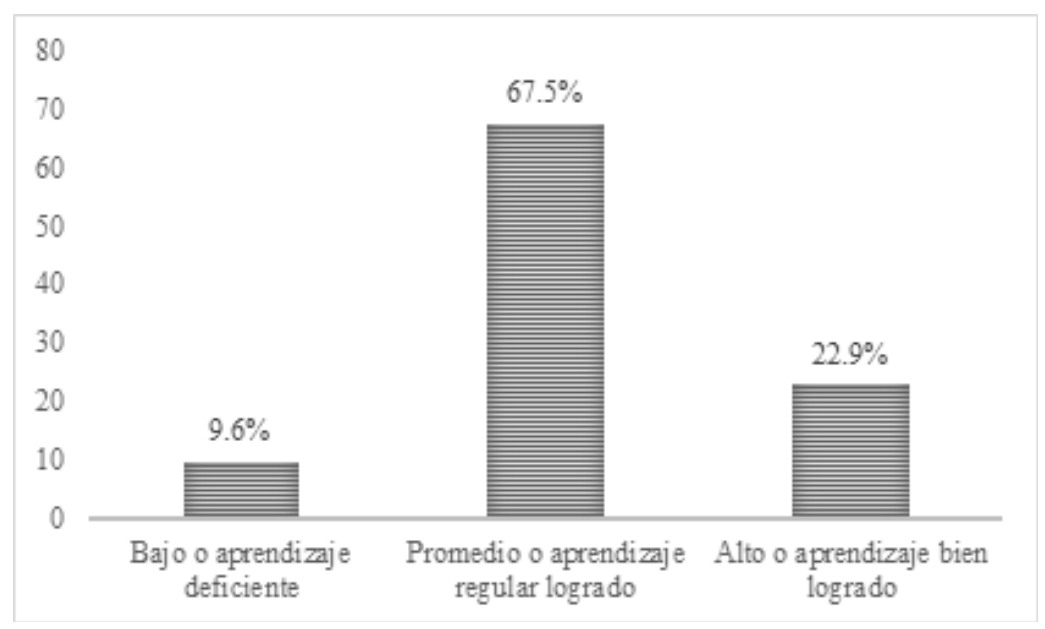

Figura 8. Niveles de rendimiento académico en general

De acuerdo a la tabla 2, el coeficiente de correlación de Rho de Spearman para actividad física y deporte, y el rendimiento académico es $r=0.8019$, con un grado de correlación positiva considerable, según los índices de correlación que propone Hernández et al. (2014, p. 305 ). Es una correlación significativa puesto que $\mathrm{p}=0.00$ es menor al nivel de significancia de 0.05 . 
Tabla 2.

Correlación entre actividad física y deporte, y el rendimiento académico

\begin{tabular}{llcc}
\hline & & $\begin{array}{c}\text { Actividad física - de- } \\
\text { porte }\end{array}$ & $\begin{array}{l}\text { Rendimiento acadé- } \\
\text { mico }\end{array}$ \\
\hline \multirow{3}{*}{$\begin{array}{l}\text { Actividad física y de- } \\
\text { porte }\end{array}$} & Correlación de Rho de Spear- & 1 & $0.8019(* *)$ \\
& man & & .000 \\
& Sig. (bilateral) & 157 & 157 \\
& $\mathrm{~N}$ & $0.8019(* *)$ & 1 \\
& Correlación de Rho de Spear- & .000 \\
Rendimiento académico & Sig. (bilateral) & 157 & 157 \\
& $\mathrm{~N}$ & &
\end{tabular}

De acuerdo a la tabla 3, el coeficiente de correlación de Rho de Spearman para recreación y manejo del tiempo libre, y el rendimiento académico es $\mathrm{r}=0.7386$, con un grado de correlación positiva media. Es una correlación significativa puesto que $\mathrm{p}=0.00$ es menor al nivel de significancia de 0.05 .

Tabla 3.

Correlación entre recreación y manejo del tiempo libre, y el rendimiento académico

\begin{tabular}{llll}
\hline & & $\begin{array}{l}\text { Recreación y manejo del } \\
\text { tiempo libre }\end{array}$ & $\begin{array}{l}\text { Rendimiento aca- } \\
\text { démico }\end{array}$ \\
\hline \multirow{2}{*}{$\begin{array}{l}\text { Recreación y manejo del } \\
\text { tiempo libre }\end{array}$} & $\begin{array}{l}\text { Correlación de Rho de } \\
\text { Spearman }\end{array}$ & 1 & $0.7386(* *)$ \\
& Sig. (bilateral) & & .000 \\
& $\mathrm{~N}$ & 157 & 157 \\
& Correlación de Rho de & $0.7386(* *)$ & 1 \\
Rendimiento académico & Spearman & .000 & \\
& Sig. (bilateral) & 157 & 157 \\
\hline
\end{tabular}

** La correlación es significativa al nivel 0,01 (bilateral)

De acuerdo a la tabla 4, el coeficiente de correlación de Rho de Spearman para autocuidado y cuidado médico, y el rendimiento académico es $\mathrm{r}=0.7425$, con un grado de correlación positiva media. Es una correlación significativa puesto que $\mathrm{p}=0.00$ es menor al nivel de significancia de 0.05 .

Tabla 4. Correlación entre autocuidado y cuidado médico, y el rendimiento académico

\begin{tabular}{llll}
\hline & & $\begin{array}{l}\text { Autocuidado - cuidado } \\
\text { médico }\end{array}$ & $\begin{array}{l}\text { Rendimiento acadé- } \\
\text { mico }\end{array}$ \\
\hline \multirow{3}{*}{$\begin{array}{l}\text { Autocuidado y cuidado } \\
\text { médico }\end{array}$} & Correlación de Rho de Spe- & 1 & $0.7425(* *)$ \\
& $\begin{array}{l}\text { arman } \\
\text { Sig. (bilateral) }\end{array}$ & 157 & .000 \\
& $\mathrm{~N}$ & $0.7425(* *)$ & 157 \\
& Correlación de Rho de Spe- & 1 \\
& arman & .000 & 157 \\
& Sig. (bilateral) & 157 &
\end{tabular}

** La correlación es significativa al nivel 0,01 (bilateral). 
De acuerdo a la tabla 5, el coeficiente de correlación de Rho de Spearman para hábitos alimenticios y el rendimiento académico es $\mathrm{r}=0.5599$, con un grado de correlación positiva media. Es una correlación significativa puesto que $\mathrm{p}=0.00$ es menor al nivel de significancia de 0.05 .

Tabla 5.

Correlación entre hábitos alimenticios y el rendimiento académico

\begin{tabular}{llll}
\hline & & Hábitos alimenticios & Rendimiento académico \\
\hline \multirow{4}{*}{ Hábitos alimenticios } & Correlación de Rho de Spearman & 1 & $0.5599(* *)$ \\
& Sig. (bilateral) & & .000 \\
& $\mathrm{~N}$ & 157 & 157 \\
& Correlación de Rho de Spearman & $0.5599(* *)$ & 1 \\
\multirow{2}{*}{ Rendimiento académico } & Sig. (bilateral) & .000 & 157 \\
& $\mathrm{~N}$ & 157 & 157 \\
& $* *$ &
\end{tabular}

** La correlación es significativa al nivel 0,01 (bilateral)

De acuerdo a la tabla 6, el coeficiente de correlación de Rho de Spearman para consumo de alcohol, tabaco y otras drogas y el rendimiento académico es $\mathrm{r}=0.5321$, con un grado de correlación positiva media. Es una correlación significativa puesto que $\mathrm{p}=0.00$ es menor al nivel de significancia de 0.05 .

Tabla 6.

Correlación entre consumo de alcohol, tabaco y otras drogas, y el rendimiento académico

\begin{tabular}{llll}
\hline & & $\begin{array}{l}\text { Consumo de alcohol, tabaco } \\
- \text { otras drogas }\end{array}$ & $\begin{array}{l}\text { Rendimiento aca- } \\
\text { démico }\end{array}$ \\
\hline \multirow{3}{*}{$\begin{array}{l}\text { Consumo de alcohol, tabaco y } \\
\text { otras drogas }\end{array}$} & $\begin{array}{l}\text { Correlación de Rho de } \\
\text { Spearman }\end{array}$ & 1 & $0.5321(* *)$ \\
& $\begin{array}{l}\text { Sig. (bilateral) } \\
\text { Rendimiento académico }\end{array}$ & & .000 \\
& $\mathrm{~N}$ & 157 & 157 \\
& Correlación de Rho de & $0.5321(* *)$ & 1 \\
& Spearman & .000 & \\
& Sig. (bilateral) & 157 & 157 \\
\hline
\end{tabular}

** La correlación es significativa al nivel 0,01 (bilateral)

De acuerdo a la tabla 7, el coeficiente de correlación de Rho de Spearman para hábitos de sueño y el rendimiento académico es $\mathrm{r}=0.8270$, con un grado de correlación positiva considerable. Es una correlación significativa puesto que $\mathrm{p}=0.00$ es menor al nivel de significancia de 0.05 .

Tabla 7. Correlación entre hábitos de sueño y el rendimiento académico

\begin{tabular}{llll}
\hline & & Hábitos de sueño & Rendimiento académico \\
\hline \multirow{2}{*}{ Hábitos de sueño } & Correlación de Rho de Spearman & 1 & $0.8270(* *)$ \\
& Sig. (bilateral) & & .000 \\
& $\mathrm{~N}$ & 157 & 157 \\
& Correlación de Rho de Spearman & $0.8270(* *)$ & 1 \\
Rendimiento académico & Sig. (bilateral) & .000 & \\
& $\mathrm{~N}$ & 157 & 157 \\
\hline
\end{tabular}

** La correlación es significativa al nivel 0,01 (bilateral) 
De acuerdo a la tabla 8, el coeficiente de correlación de Rho de Spearman para estilos de vida saludable y rendimiento académico es $\mathrm{r}=0.6529$, entonces, con un grado de correlación positiva media. Es una correlación significativa puesto que $\mathrm{p}=0.00$ es menor al nivel de significancia de 0.05 .

Tabla 8. Correlación entre estilos de vida saludable y rendimiento académico

\begin{tabular}{llll}
\hline & & $\begin{array}{l}\text { Estilos de vida salu- } \\
\text { dable }\end{array}$ & $\begin{array}{l}\text { Rendimiento acadé- } \\
\text { mico }\end{array}$ \\
\hline \multirow{2}{*}{$\begin{array}{l}\text { Estilos de vida saluda- } \\
\text { ble }\end{array}$} & Correlación de Rho de Spear- & 1 & $0.6529(* *)$ \\
& man & & .000 \\
& Sig. (bilateral) & 157 & 157 \\
& $\mathrm{~N}$ & 157 & 1 \\
& Correlación de Rho de Spear- & $0.6529(* *)$ & \\
& man & .000 & 157 \\
\hline & Sig. (bilateral) & 157 &
\end{tabular}

\section{DISCUSIÓN Y CONCLUSIONES}

La mayoría de estudiantes en estilos de vida saludable (85.99\%) tienen un estilo medianamente saludable y en rendimiento académico (67.50\%) tienen un nivel promedio o aprendizaje regular logrado, y en menores porcentajes de estudiantes en estilos de vida saludable $(14.01 \%)$ tienen un estilo saludable, y en menores porcentaje de estudiantes en rendimiento académico (22.9\%) tienen un nivel alto o aprendizaje bien logrado, y sólo un $9.60 \%$ alcanzan un nivel bajo en rendimiento académico o aprendizaje deficiente. Esto indica que la presencia de estilos de vida medianamente saludable en los estudiantes favorece el rendimiento académico en un nivel promedio o aprendizaje regular logrado.

Existe un coeficiente de correlación de Rho de Spearman de $\mathrm{r}=0.8019$ entre las variables actividad física y deporte, y rendimiento académico, con un grado de correlación positiva considerable y significativa, significa cuanto mejor estilo saludable en actividad física y deporte tengan los estudiantes mejor será su nivel de rendimiento académico o aprendizaje, pues la actividad la actividad física y deporte recreativo no competitivo y sin esfuerzo, ayuda a liberar serotonina, reduce la ansiedad y tensión, mejora el autocontrol y regulación emocional, reduce la depresión y aumenta la sensación de bienestar para que los estudiantes puedan planificar mejor y con agrado sus estudios (Lema et al., 2008, p.73).

Existe un coeficiente de correlación de Rho de Spearman de $\mathrm{r}=0.8270$ entre hábitos de sueño y el rendimiento académico, con un grado de correlación positiva considerable y significativa, es decir, cuanto mejor estilo saludable en hábitos de sueño tengan los estudiantes mejor será su rendimiento académico o aprendizaje, pues dormir bien y lo necesario contribuye a que el estudiante recupere las energías para que pueda volver a emprender mejor sus actividades académicas, el sueño restaura las funciones corporales, mentales, fisiológicas y bioquímicas que constituye una necesidad que tiene que ser satisfecha en calidad y cantidad (Lema et al., 2008, p. 74).

También se obtuvo un coeficiente de correlación de Rho de Spearman de $\mathrm{r}=0.7386$ entre recreación y manejo del tiempo libre, y el rendimiento académico, con un grado de correlación positiva media y significativa, quiere decir, cuanto mejor estilo saludable en recreación 
y manejo del tiempo libre tengan los estudiantes mejor será su rendimiento académico o aprendizaje: Pues los estudiantes deben elegir sus actividades libremente para satisfacer su necesidades personales, tener un tiempo para el descanso, la diversión, la recreación y el desarrollo personal, esto va generar satisfacción personal y tranquilidad en las tareas académicas de los estudiantes (Lema et al., 2008, p.73).

Asimismo, se obtuvo un coeficiente de correlación de Rho de Spearman de $r=0.7425$ entre autocuidado y cuidado médico, y rendimiento académico, con un grado de correlación positiva media y significativa. Pues el autocuidado personal mantiene la vida, la salud y el bienestar y esto genera seguridad en los estudiantes para poder seguir con sus estudios, y seguir sus actividades habituales personales y de grupo (Carrillo y Amaya, 2006, p.43).

También se obtuvo un coeficiente de correlación de Rho de Spearman de $r=0.5599$ entre hábitos alimenticios y el rendimiento académico, y un coeficiente de correlación de Rho de Spearman de $\mathrm{r}=0.5321$ entre el consumo de alcohol, tabaco y otras sustancias, y el rendimiento académico, ambas correlaciones con grado de correlación positiva media y significativa. Pues los hábitos adecuados de alimentación favorecen la recuperación de energías y el restablecimiento de organismo para seguir con nuestras actividades cotidianas, y el control en el consumo de alcohol va generar un buen estado salud propicio para el aprendizaje de los estudiantes (Carrillo \& Amaya, 2006, p.35).

Finalmente, existe una correlación de Rho de Spearman de $\mathrm{R}=0.6529$ entre estilos de vida saludable y rendimiento académico, con un grado de correlación positiva media significativa, lo que indica que cuanto más saludable sean los estilos de vida, mejor serán las condiciones para el aprendizaje de los estudiantes (Evaristo, 2012, p. 45).

\section{REFERENCIAS}

Arrivillaga, M. \& Salazar, I.C. (2005). Creencias relacionadas con el estilo de vida de jóvenes latinoamericanos. Psicología Conductual, 13(1), 19-36 http://proinapsa.uis.edu.co/redcups/Biblioteca/Documentos\%20REDCUPS/Investigacion_Creencias_y_\%20Estilos_de_vida.pdf

Arrivillaga, M., Salazar, I.C. \& Correa, D. (2003). Creencias sobre la salud y su relación con las prácticas de riesgo o de protección en jóvenes universitarios. Colombia Médica, 34(4), 186195. https://www.redalyc.org/pdf/283/28334403.pdf

Carrillo, H. O., \& Amaya, M. C. (2006). Estilos de vida saludables de las enfermeras (os) docentes de la Escuela de Enfermería de la Universidad Pedagógica y Tecnológica de Colombia. Biblioteca Las Casas, 2(2). http://www.index-f.com/lascasas/documentos/lc0128.pdf

Evaristo, T. A. (2012). Calidad de vida y su relación con el rendimiento académico según características sociodemográficas de estudiantes de Odontología [Tesis de maestría, UNMSM, Lima]. http://cybertesis.unmsm.edu.pe/bitstream/cybertesis/2293/1/evaristo_ct.pdf

Hernández, R., Fernández, C., \& Baptista, P. (2014) Metodología de la investigación. (6ta.Ed.). McGraw-Hi$11 /$ Interamericana Editores, S.A. de C.V.

Kerlinger, F. N. \& Lee, H.B. (2002). Investigación del comportamiento. Cuarta edición. McGraw-Hill/Interamericana.

Lema, L.F.; Salazar, I.C.; Varela, M.T.; Tamayo, J.A.; Rubio, A. y Botero, A. (2008). Comportamiento y salud de los jóvenes universitarios: satisfacción con el estilo de vida.

Pensamiento Psicológico, 5(12), 71-78. http://portales.puj.edu.co/psicorevista/components/com_joomlib/ ebooks/PS12-5.pdf 